\title{
A Multiscale Approach to Contour Detection by Texture Suppression
}

\author{
Giuseppe Papari*, Patrizio Campisi ${ }^{\S * *}$, Nicolai Petkov*, and Alessandro Neri** \\ *Institute of Mathematics and Computing Science, University of Groningen, \\ P. O. Box 800, 9700 AV Groningen, The Netherlands \\ E-mail: petkov@cs.rug.nl \\ **Dipartimento di Elettronica Applicata, Università degli Studi di Roma "Roma Tre", \\ Via della Vasca Navale 84, 00146 Roma, Italy \\ Ph.: +39.06.55177064, Fax: +39.06.55177026, E-mail: campisi@uniroma3.it
}

\begin{abstract}
In this paper we propose a multiscale biologically motivated technique for contour detection by texture suppression. Standard edge detectors reacts to all the local luminance changes, irrespective whether they are due to the contours of the objects represented in the scene, rather than to natural texture like grass, foliage, water, etc. Moreover, edges due to texture are often stronger than edges due to true contours. This implies that further processing is needed to discriminate true contours from texture edges. In this contribution we exploit the fact that, in a multiresolution analysis, at coarser scales, only the edges due to object contours are present while texture edges disappear. This is used in combination with surround inhibition, a biologically motivated technique for texture suppression, in order to build a contour detector which is insensitive to texture. The experimental results show that our approach is also robust to additive noise.
\end{abstract}

Keywords: Contour detection, edge, multiscale analysis, surround inhibition, morphological analysis, texture

\section{INTRODUCTION}

Contour detection is considered a fundamental operation in image processing and computer vision, with a large number of studies published in the last two decades. In the framework of this paper, the term contour is used to represent a "line delimiting a (part of an) object in a scene". This is a more sophisticated concept than that of an edge, which refers to a "not negligible local luminance change". Therefore, in our approach, contour detection is a global concept related to the recognition of meaningful objects.

Examples of edge detectors are operators that incorporate linear filtering [1, 2, 3], local orientation analysis [4], fitting of analytical models to the image data $[5,6]$ and local energy $[7,8]$. Since these operators do not make any difference between various types of edges, such as texture edges vs. object contours and region boundaries, they are known as noncontextual or, simply, general edge detectors [9].

Other studies propose more elaborate edge detection techniques that take into account additional information around an edge, such as local image statistics, image topology, perceptual differences in local cues (e.g. texture, color), edge continuity and density, etc. Examples are dual frequency band analysis [10], anisotropic diffusion [11, 12], use of edge density information [13] and biologically motivated surround modulation $[14,15,16]$. These operators are not aimed at detecting all luminance changes in an image but rather at selectively enhancing only those edges that are of interest in the context of a specific task, such as the outlines of tissues in medical images, object contours in natural image scenes, boundaries between different texture regions, etc. Such methods are usually referred to as contextual edge detectors.

From a perceptual point of view, in its early stages the human visual system differentiates between isolated edges, such as object contours and region boundaries, on the one hand, and edges in a group, such as those in texture, on the other. Various psychophysical studies have shown that the perception of an oriented stimulus, e.g. a line segment, can be influenced by the presence of other such stimuli (distractors) in its neighborhoods [25 - 27]. Neurophysiological studies 
show that the response of an orientation selective visual neuron to an optimal bar stimulus in its receptive field is reduced by the addition of other oriented stimuli to the surround $[28,29]$. Neurophysiologists refer to this effect as non-classical receptive field inhibition [28, 29] or surround suppression [17]. In [13], it was suggested that the biological utility of surround suppression is contour enhancement in natural images rich in background texture. In the current study we combine the principle of surround inhibition [13, 15, 18], briefly recalled in Section 2, with a multiscale approach [10] described in Section 3. The effectiveness of the proposed contour detection method is demonstrated in Section 4, where it is also shown to be robust to both local texture and noise. We draw conclusions in Section 5.

\section{SINGLE SCALE CONTOUR DETECTION WITH SURROUND INHIBITION}

Here we recall the surround inhibition technique for a single scale and in the next section we incorporate it in a multiscale scheme. The method we propose stems from the approach described in $[13,15,18]$ where an operator that includes surround inhibition for enhanced contour detection is introduced. This approach relies on the observation that if edges are close to each other, they are likely to be due to texture. On the contrary, true contours lead to isolated edges. Within this framework, we compute a local average of the gradient intensity on a ring around each point of the image. Such term will be low when evaluated on points belonging to isolated edges, whereas it will assume a high value when evaluated on texture points. Therefore, we use this information to inhibit the edges due to texture, preserving the ones due to true contours.

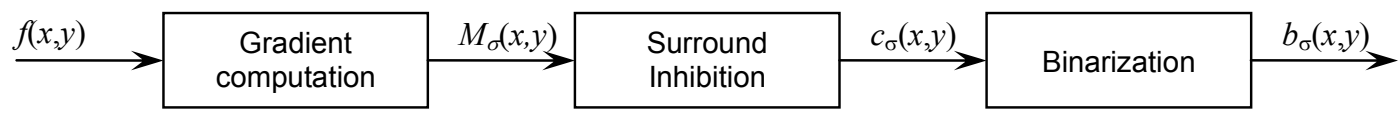

(a)

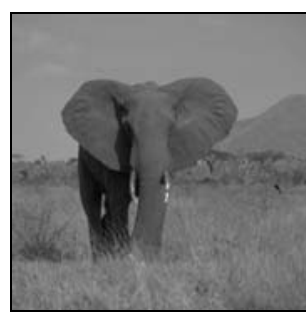

(b)

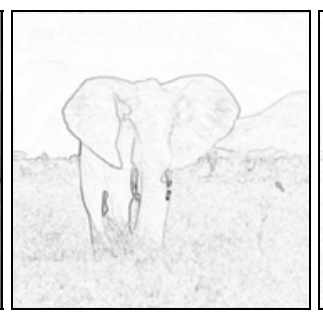

(c)

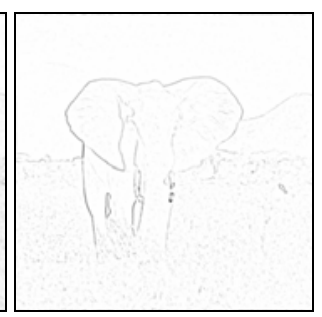

(d)

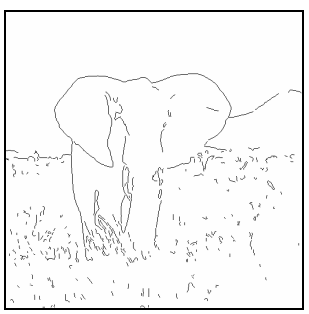

(e)

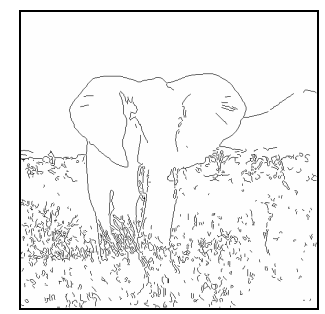

$(f)$

Fig. 1. Contour detector improved by surrounding inhibition. (a) Computation scheme. (b) Original image $f(x, y)$. (c) Magnitude $\mathrm{M}_{\sigma}(x, y)$ of the scale-dependent gradient of $f(x, y) .(d)$ Output $c(x, y)$ of the surrounding inhibition step; while contours are preserved, the activity due to grass texture is reduced. (e) Binary contour map $b(x, y)$ obtained by hysteretic thresholding and non-maximal suppression of $c(x, y) .(f)$ Binary map that is obtained by skipping the surround inhibition step, i.e. by binarizing directly $M_{\sigma}(x, y)$.

In more detail, with reference to Fig. 1, given an image $f(x, y)$, its edge map is evaluated by means of a scale dependent gradient $M_{\sigma}(x, y)$, obtained by convolving the image $f(x, y)$ with the $x$ and $y$ derivatives of a Gaussian kernel [1]:

$$
M_{\sigma}(x, y)=\sqrt{\left[\nabla_{x} F_{\sigma}(x, y)\right]^{2}+\left[\nabla_{y} F_{\sigma}(x, y)\right]^{2}}
$$

where:

$$
\left\{\begin{array}{l}
\nabla_{x} F_{\sigma}(x, y)=\left\{f * \frac{\partial g_{\sigma}}{\partial x}\right\}(x, y) \\
\nabla_{y} F_{\sigma}(x, y)=\left\{f * \frac{\partial g_{\sigma}}{\partial y}\right\}(x, y)
\end{array}, \quad g_{\sigma}(x, y)=\frac{1}{2 \pi \sigma^{2}} e^{-\frac{x^{2}+y^{2}}{2 \sigma^{2}}}\right.
$$


In order to improve the performance of the edge detector, a surround inhibition operator that takes into account the context influence of a ring area surrounding a given point is introduced in $[13,15,18]$. More specifically, let us consider the difference $\operatorname{DoG}_{\sigma}(x, y)$ of two Gaussian functions that defines a ring region centered at the point of interest $(x, y)$.

$$
\operatorname{DoG}_{\sigma}(x, y)=\left|\frac{1}{2 \pi(4 \sigma)^{2}} \exp \left(-\frac{x^{2}+y^{2}}{2(4 \sigma)^{2}}\right)-\frac{1}{2 \pi \sigma^{2}} \exp \left(-\frac{x^{2}+y^{2}}{2 \sigma^{2}}\right)\right|^{+} ; \quad w_{\sigma}(x, y)=\frac{\operatorname{DoG}_{\sigma}(x, y)}{\iint_{R^{2}} \operatorname{DoG}_{\sigma}(x, y) d x d y}
$$

where $|.|^{+}$denotes half-wave rectification:

$$
|\xi|^{+}= \begin{cases}\xi, & \xi \geq 0 \\ 0, & \xi<0\end{cases}
$$

The suppression term is obtained as follows:

$$
t_{\sigma}(x, y)=\left\{M_{\sigma} * w_{\sigma}\right\}(x, y)
$$

The output of the surrounding inhibition block is given by

$$
c_{\sigma}(x, y)=\left|M_{\sigma}(x, y)-\alpha T_{\sigma}(x, y)\right|^{+}
$$

where $\alpha$ controls the strength of the inhibition of the surroundings of the point $(x, y)$. If there is no texture in the surroundings of a point $(x, y)$, then the output $c_{\sigma}(x, y)$ of the surround suppression step is equal to its input, the gradient magnitude response $M_{\sigma}(x, y)$. If many edges are present in the surroundings of $(x, y)$, the suppression term $t_{\sigma}(x, y)$ may become so strong to cancel the magnitude response.

Finally, binary contours $b_{\sigma}(x, y)$ are extracted by using hysteretic thresholding and non-maxima suppression, similar to the way this is done in the Canny edge detector [1]. The operator that gives $b_{\sigma}(x, y)$ from the original image $f(x, y)$ will be referred as $\operatorname{SSCD}_{\sigma}$ (Single Scale Contour Detector):

$$
b_{\sigma}(x, y)=\operatorname{SSCD}_{\sigma}\{f(x, y)\}
$$

\section{MULTISCALE APPROACH}

In this Section, a multiscale approach is used in conjunction with the classical Canny edge detector (Section 3.1). Then, in Section 3.2, its combination with surround inhibition is outlined.

\subsection{Multiscale Canny edge detection}

As well known from multiresolution wavelet analysis [24], at coarser scales, the details of the image disappear and only the general morphology is present. This suggests that texture can be drastically reduced by exploiting the fact that it is present only at the finest scale, while the true contours are present also at coarser resolutions. This is illustrated in Fig. 2 where Figs $2 a$ and $2 b$ show the binary contour maps $b_{1}(x, y)$ and $b_{2}(x, y)$ obtained by the standard Canny edge detector with $\sigma=1$ and $\sigma=2$ respectively. When the coarser scale $(\sigma=2)$ is used, some texture is removed, but the contours are not well localized, shifted [20] and smoothed, and the junctions are destroyed by the non-maxima suppression [19].

In order to overcome this problem, we propose to combine the information at both scales $\sigma=1$ and $\sigma=2$ as follows: first, we perform a morphological dilation $[21,22]$ by a disk $D_{3}$ with radius of three pixel as structuring element:

$$
b_{2, U P}=b_{2} \oplus D_{3}
$$


Then we compute the binary (AND) superposition of $b_{2, U P}(x, y)$ and $b_{1}(x, y)$ (Fig. $\left.2 c\right)$. The true contours, which are detailed and well localized in $b_{1}(x, y)$, are also contained in $b_{2, U P}(x, y)$. In contrast, most of the texture present in $b_{1}(x, y)$ is not present in $b_{2, U P}(x, y)$. Consequently, the logic AND between $b_{1}(x, y)$ and $b_{2, U P}(x, y)$, shown in Fig. $2 d$, has the well detailed contours as in $b_{1}(x, y)$, whereas the texture that is not present in $b_{2, U P}(x, y)$ has been removed. Furthermore, the morphological dilation restores the junctions which have been destroyed in $b_{2}(x, y)$.

The method can be refined by removing the residual texture still present in the result (Fig. $2 d$ ) by using more scales and by introducing a surround inhibition step, as we will show in Section 3.2.

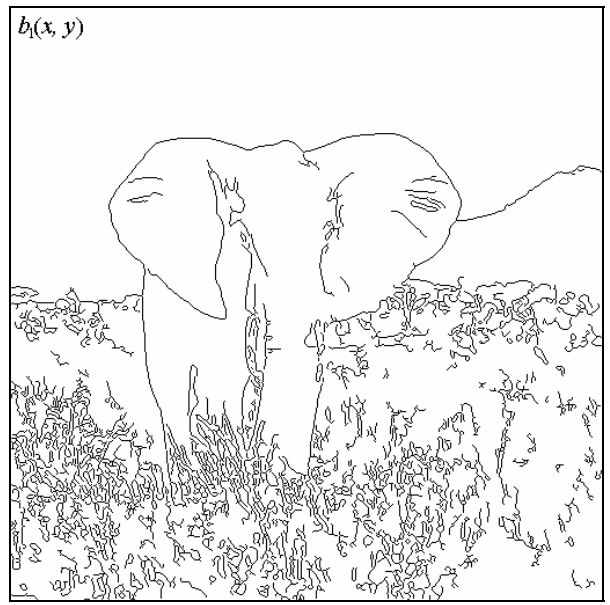

(a)

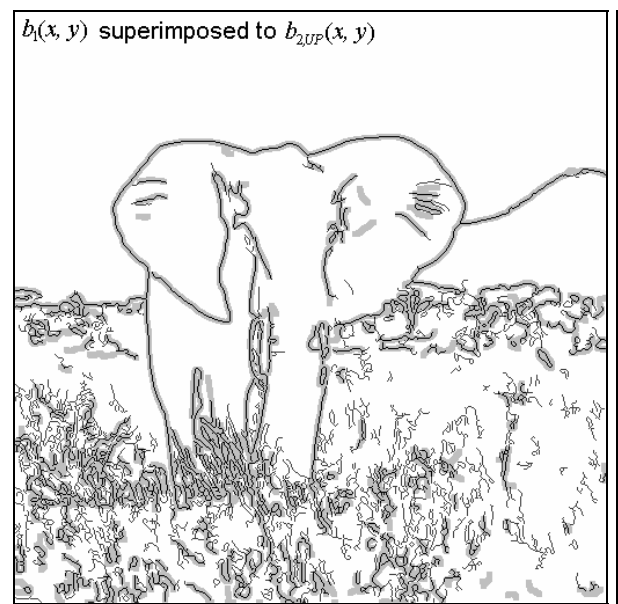

(c)

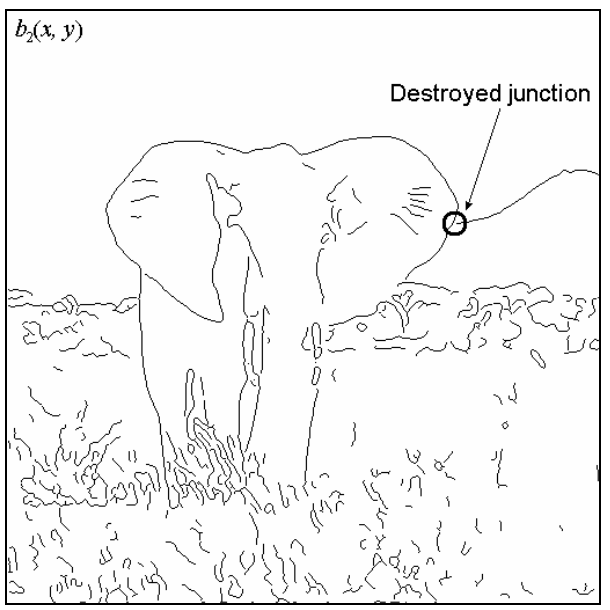

(b)

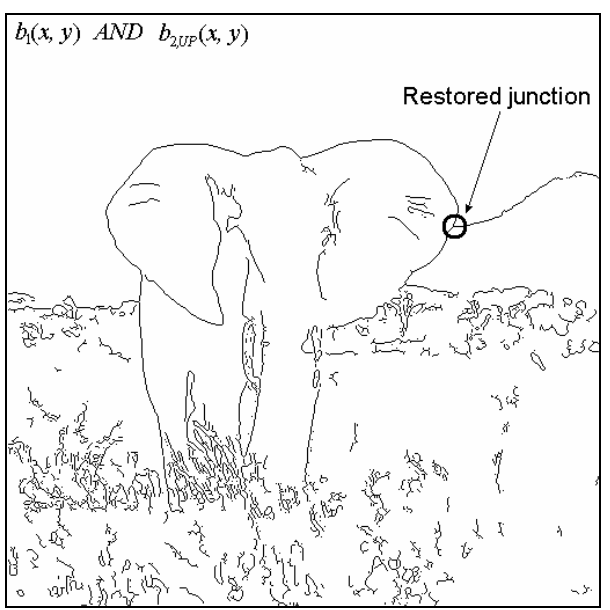

(d)

Fig. 2. $(a, b)$ Binary contour maps $b_{1}(x, y)$ and $b_{2}(x, y)$ obtained by the standard canny edge detector and hysteretic thresholding with $\sigma=1$ and $\sigma=2$ respectively. At the finer scale the borders are well detailed, but much texture is present; at coarser scale, texture is reduced but the contours are smoother, less localized and the junction are destroyed. $(c)$ Superimposition of the contour map at fine scale and the morphologically dilated contour map at coarse scale and $(d)$ the result of their logic AND; texture is reduced, contours are well detailed ant the morphological dilation restores the junction.

In order to reduce the amount of computation, in our implementation we decimate the images at the coarser scales: instead of detecting the edges in the original image with $\sigma=K$, we operate with $\sigma=1$ on the decimated image by a factor $K$ since it can be proved that no information is lost [23]. The size of each obtained contour map is then doubled before performing the morphological dilation. 
More precisely, we define a mathematical operator DTO (Doubling and Ticking Operator), which operates as shown in Fig. 3. Given a generic binary map $b(x, y)$, a second binary map $\beta(x, y)$ is obtained by doubling the coordinates of all the nonzero pixels:

$$
\beta(x, y)= \begin{cases}b\left(\frac{x}{2}, \frac{y}{2}\right), & x, y \text { even } \\ 0, & \text { otherwise }\end{cases}
$$

Then, each nonzero pixel of $\beta(x, y)$ is substituted by a disk of radius 3 pixels:

$$
\operatorname{DTO}\{b(x, y)\}=\left[\beta \oplus D_{3}\right](x, y)
$$

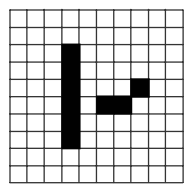

(a)

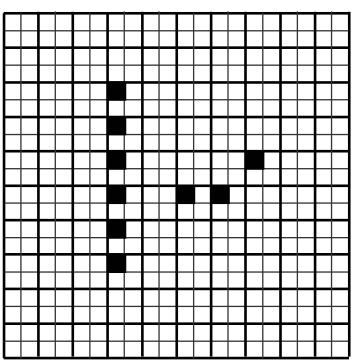

(b)

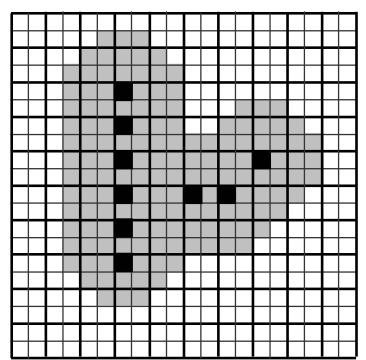

(c)

Fig. 3. (a) Initial binary contour map $b(x, y)$, with a destroyed junction. (b) Binary map $\beta(x, y)$ obtained by doubling the coordinates of all the nonzero pixels of $(a)$. (c) Result DTO $\{b(x, y)\}$ of the dilation, with the restored junction.

\subsection{Multiscale contour detection with surround inhibition}

The algorithm we propose consists in applying the process described in Section 3.1 to the binary contour maps obtained by the surround inhibition scheme of Fig. 1 at $N$ different scales. The principle scheme of the approach is given in Fig. 4 . Given the original image $f(x, y)$, decimated replicas at $N$ coarser scales, $f_{2^{n}}(x, y)$ with $n=0,1, \ldots, N-1$ are obtained:

$$
f_{2^{n}}(x, y)= \begin{cases}f(x, y), & n=0 \\ {\left[f * g_{\sigma=2^{n}}\right](x, y)_{2^{n} \downarrow},} & n=1,2, \ldots, N-1\end{cases}
$$

where the low pass filter $g_{\sigma}$ is defined by eq. (2) and the subscript $2^{n} \downarrow$ stands for "undersampling by a factor $2^{\text {n๘. }}$. Then, the contour detector described in Section 2, with $\sigma=1$, is applied to each decimated image, obtaining the binary contour maps $b_{2^{n}}(x, y)$

$$
b_{2^{n}}(x, y)=\operatorname{SSCD}_{\sigma=1}\left\{f_{2^{n}}(x, y)\right\}
$$

Starting by the coarsest scale $n=N-1$, we double the size of the image and apply the morphological dilation by the DTO operator. We multiply the dilated image by the contour map at the next finer scale and, then, we repeat the entire process up to the finest scale:

$$
\left\{\begin{array}{l}
\tilde{b}_{2^{N-1}}(x, y)=b_{2^{N-1}}(x, y) \\
\tilde{b}_{2^{n-1}}=b_{2^{n-1}}(x, y) \cdot \operatorname{DTO}\left\{\tilde{b}_{2^{n}}(x, y)\right\}, \quad n=N-2, \ldots, 1
\end{array}\right.
$$




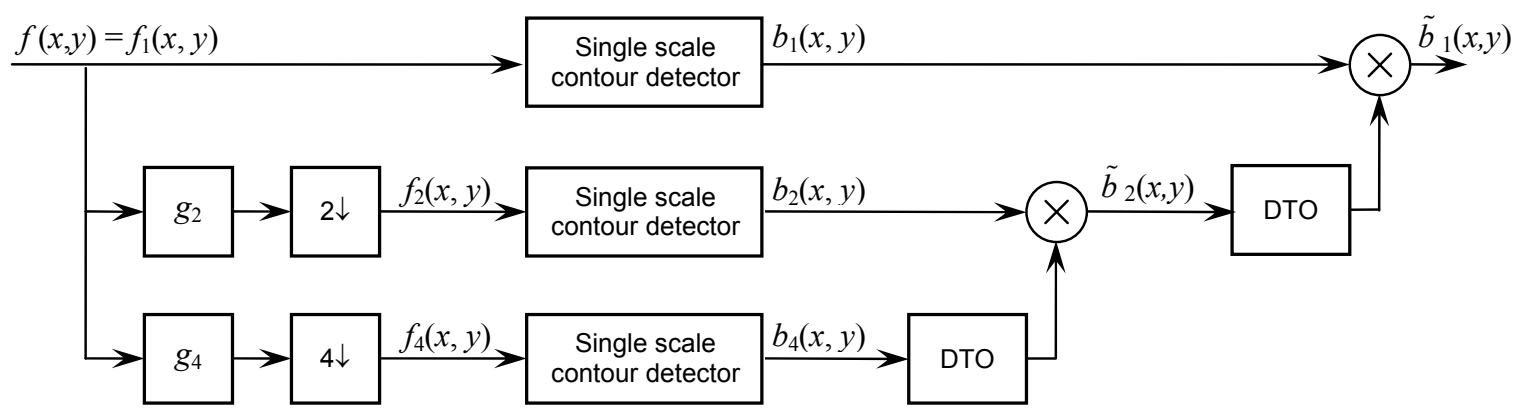

Fig. 4: Multiscale approach to contour detection by texture surround suppression.

The proposed multiscale algorithm is summarized in Table 1.

\begin{tabular}{ll}
\hline 1. Computation of the images $f_{2^{n}}(x, y)$ & (Multiscale analysis) \\
2. Computation of the binary maps $b_{2^{n}}(x, y)$ & (Single scale contour detection) \\
3. Set $\tilde{b}_{2^{N-1}}(x, y)=b_{2^{N-1}}(x, y)$ & (Initialization) \\
4. For $n=N-2$ to $n=1$ & (Scales combination) \\
$\qquad \tilde{b}_{2^{n-1}}(x, y)=b_{2^{n-1}}(x, y) \cdot \operatorname{DTO}\left\{\tilde{b}_{2^{n}}(x, y)\right\}$ & \\
End & (Output) \\
5. $\tilde{b}_{1}(x, y)$ is the final output
\end{tabular}

Table 1: Multiscale contour detection algorithm.

In Fig. 5, the images $b_{1}(x, y), b_{2}(x, y)$, and $b_{4}(x, y)$ for the test image "Elephant" are displayed. The binary edge map $b_{1}(x, y)$, at the finest scale, has well detailed contours. However, some residual texture is still present. At coarser scales, the contours become less detailed but the residual texture progressively vanishes. The surround inhibition helps in reducing the texture present in the binary contour map, still maintaining the true contours, as it is evident by comparing Figs. $5 a$ and $5 b$ with Figs. $2 a$ and $2 b$ respectively.

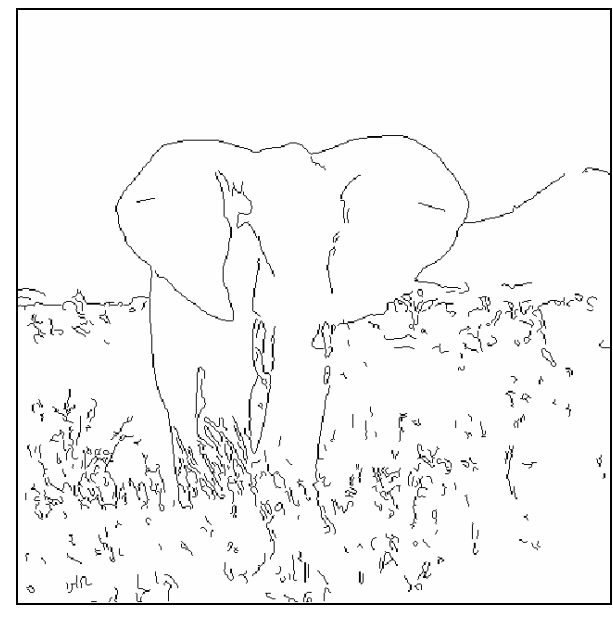

(a)

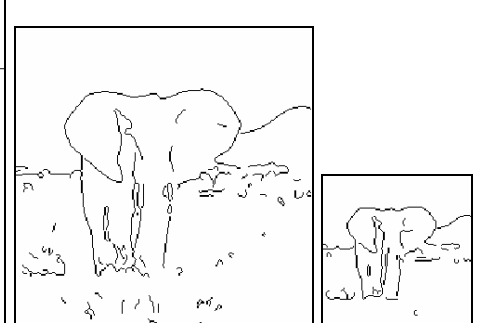

(b) (c)

Fig. 5. Binary contour maps: $(a) b_{1}(x, y)$ at the finest scale, $(b) b_{2}(x, y)$ at the medium scale, and $(c) b_{4}(x, y)$ at the coarsest scale. Compare the first two images with Figs $2 a$ and $2 b$ to see the improvement brought by the surround inhibition. 

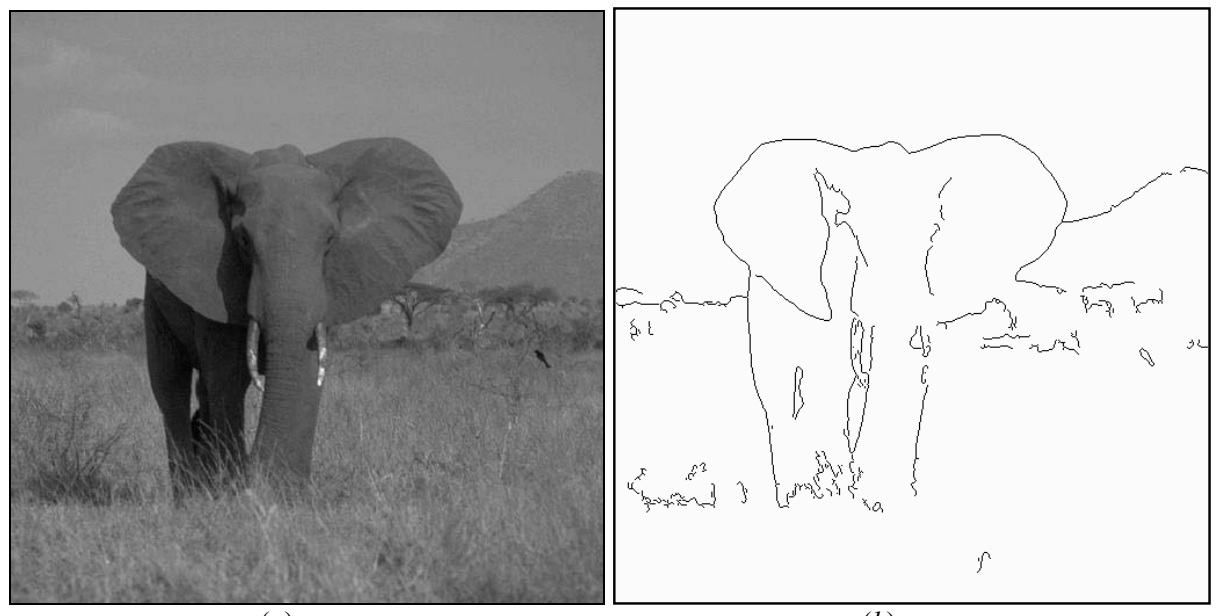

(a)

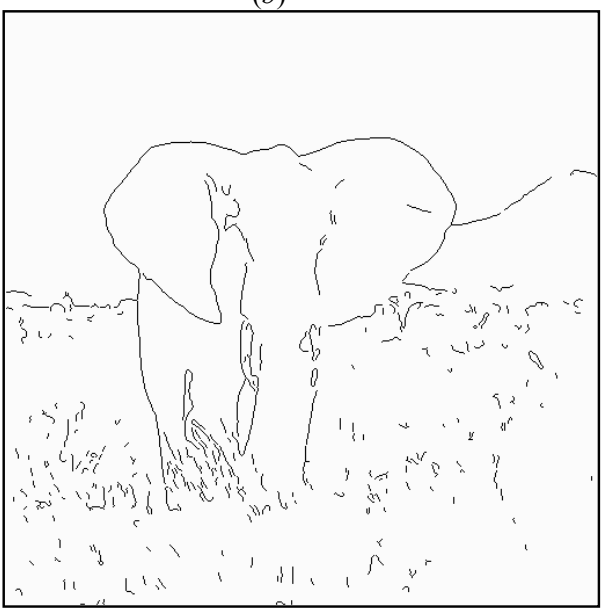

(c)

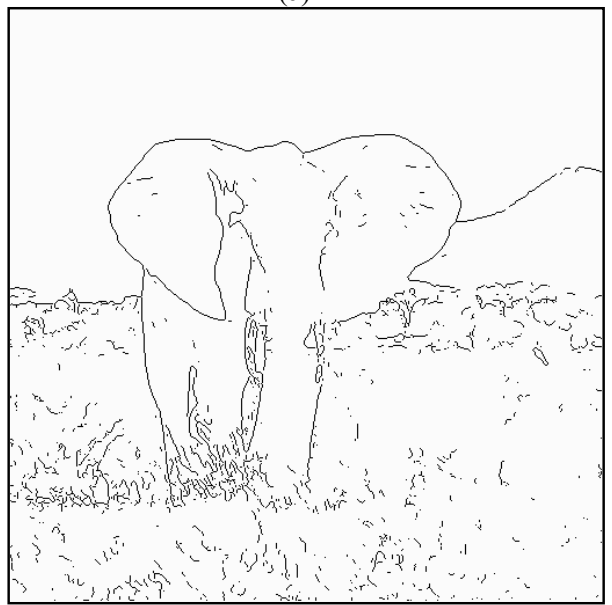

(d)

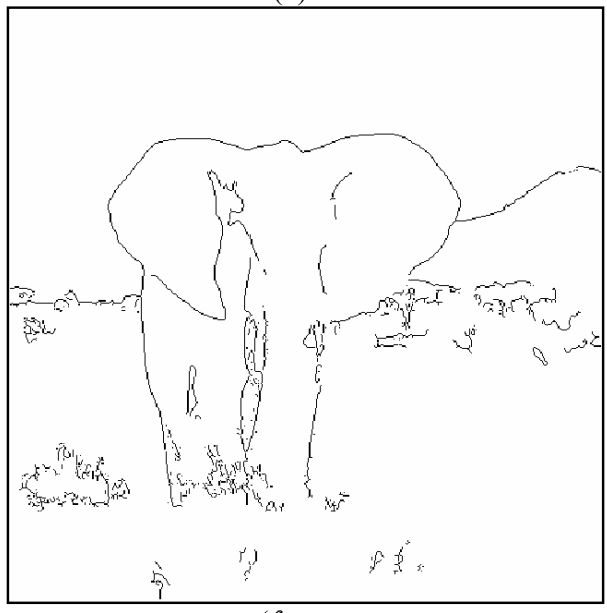

(e)

(f)

Fig. 6. (a). Original image (Elephant). (b) Contours detected using the proposed approach. (c) Result of single scale Canny edge detection. (d) Result of single scale Canny edge detection augmented with surround inhibition. (e) Result of multiscale edge detection without surround inhibition. (f) Multiscale edge detection according to the scheme of Fig.4, where the inhibition step is not performed. 


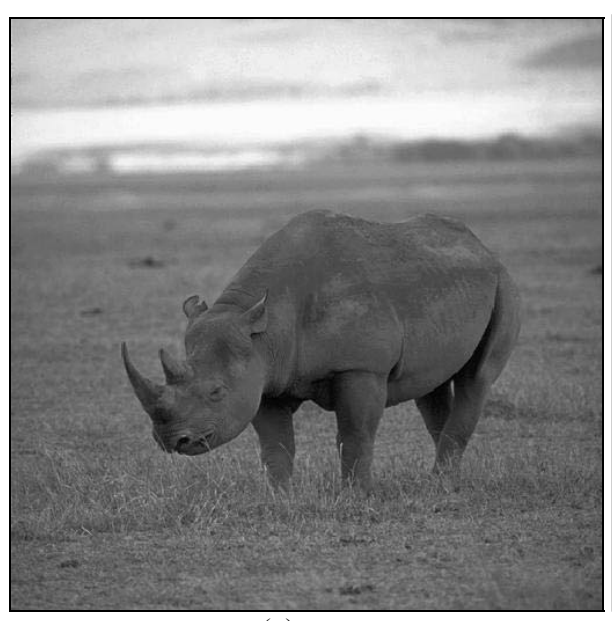

(a)

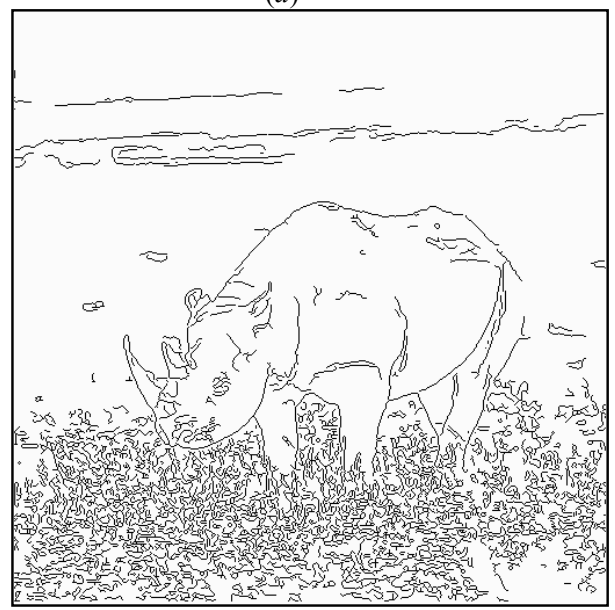

(c)

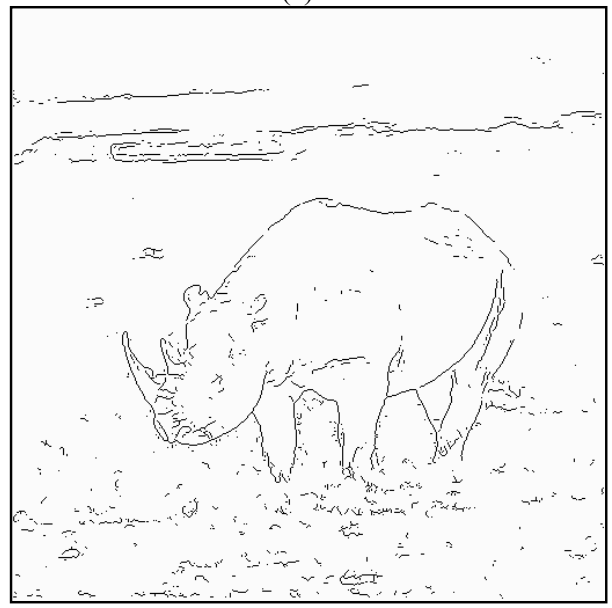

(e)

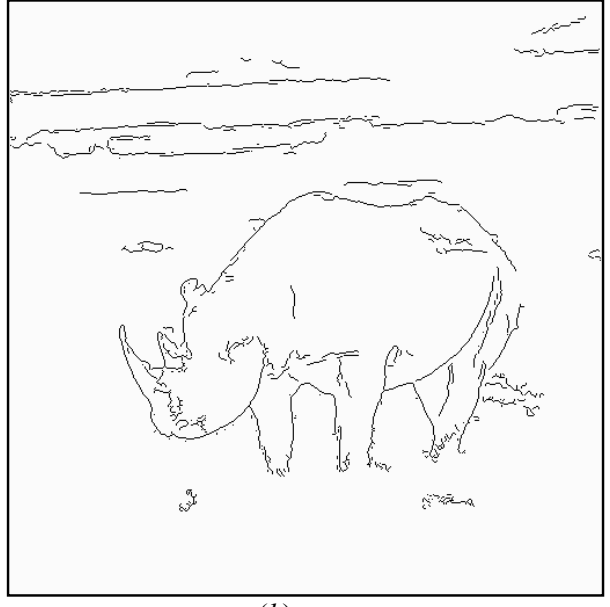

(b)

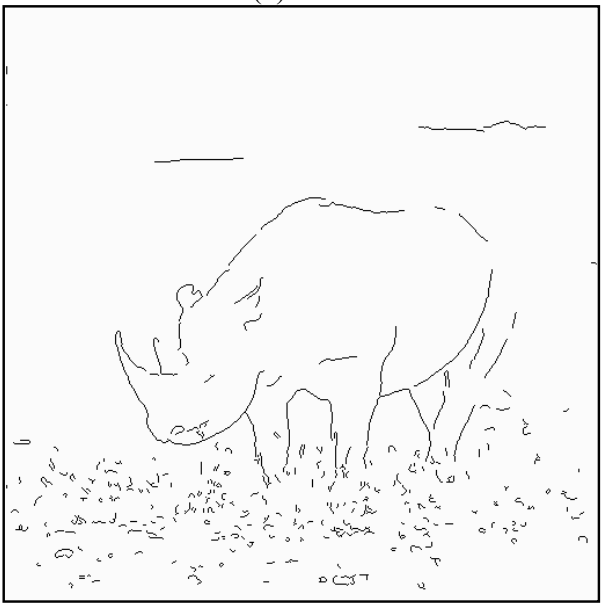

(d)

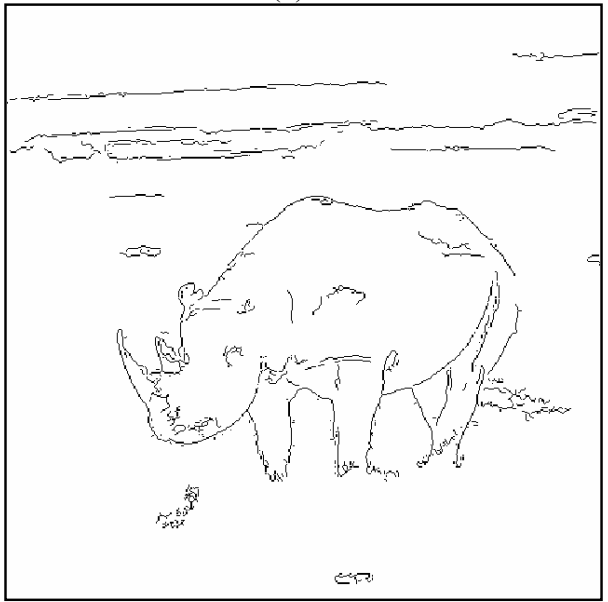

$(f)$

Fig. 7. (a). Original image (Rhino). (b) Contours detected using the proposed approach. (c) Result of single scale Canny edge detection. (d) Result of single scale Canny edge detection augmented with surround inhibition. (e) Result of multiscale edge detection without surround inhibition. (f) Multiscale edge detection according to the scheme of Fig.4, where the inhibition step is not performed. 

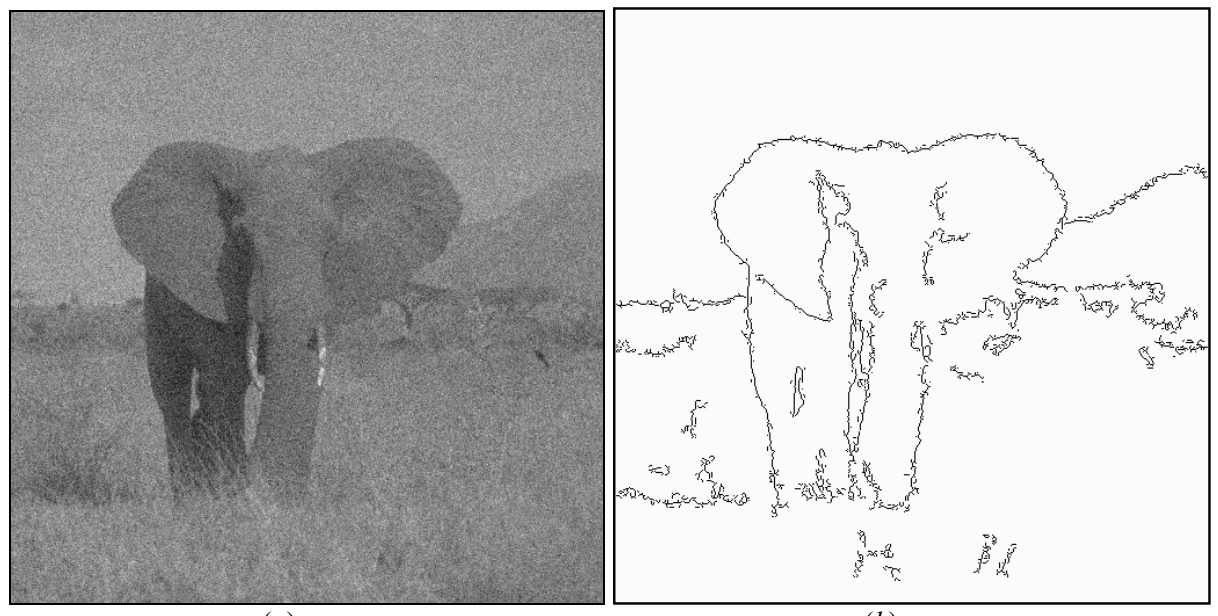

(a)

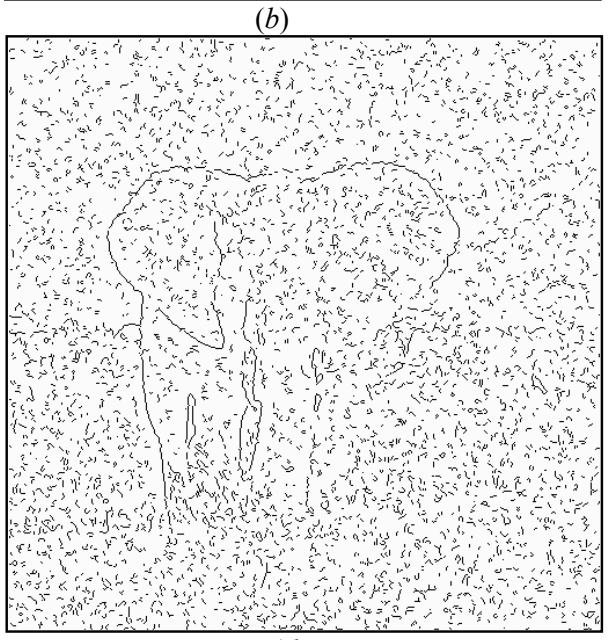

(d)

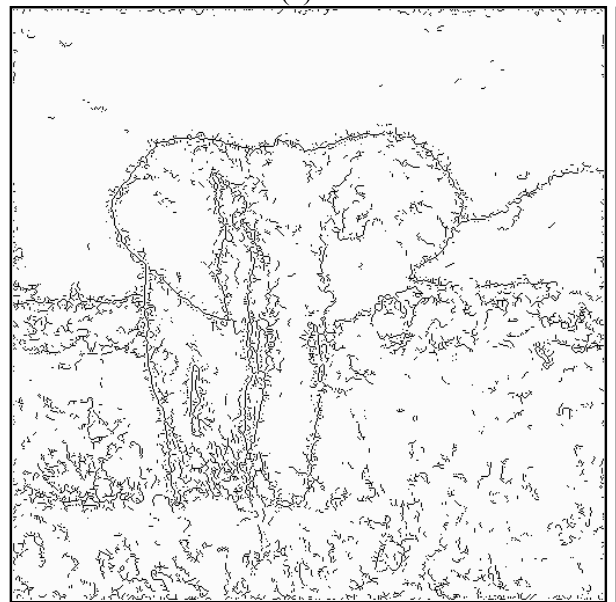

(e)

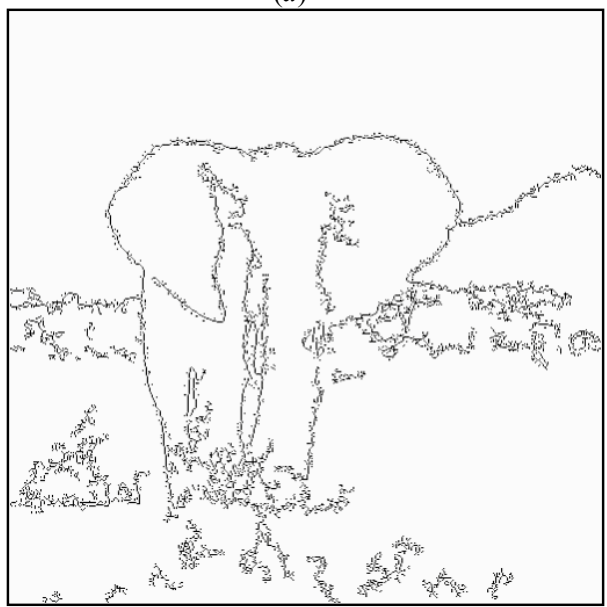

(f)

Fig. 8. (a). Input image (Elephant) contaminated by additive Gaussian noise ( $\mathrm{SNR}=13.5 \mathrm{~dB})$. (b) Contours detected using the proposed approach. (c) Result of single scale Canny edge detection. (d) Result of single scale Canny edge detection augmented with surround inhibition. (e) Result of multiscale edge detection without surround inhibition. (f) Multiscale edge detection according to the scheme of Fig.4, where the inhibition step is not performed. 


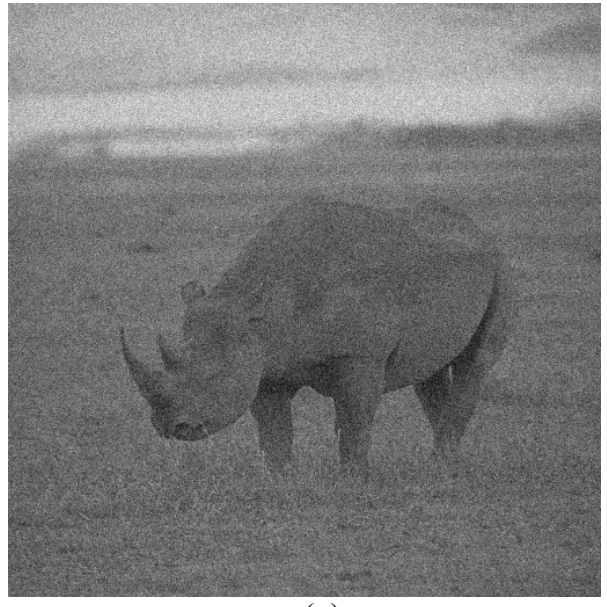

(a)

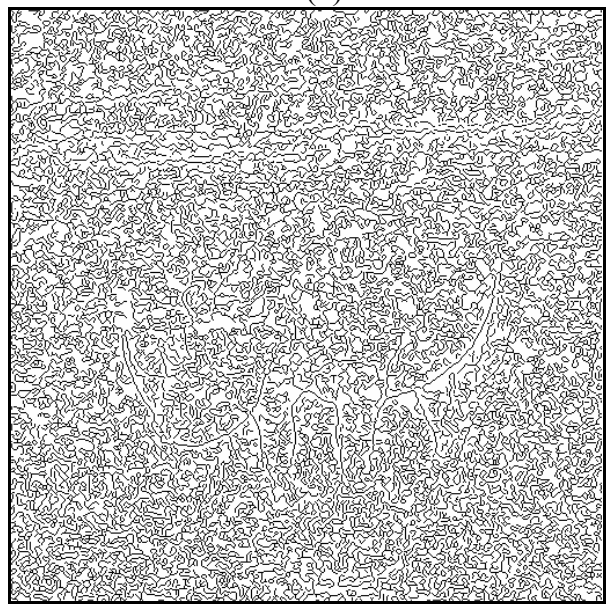

(c)

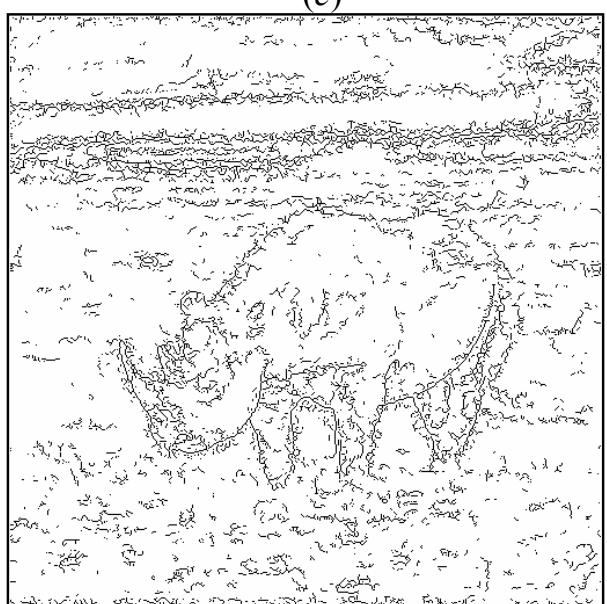

$(e)$

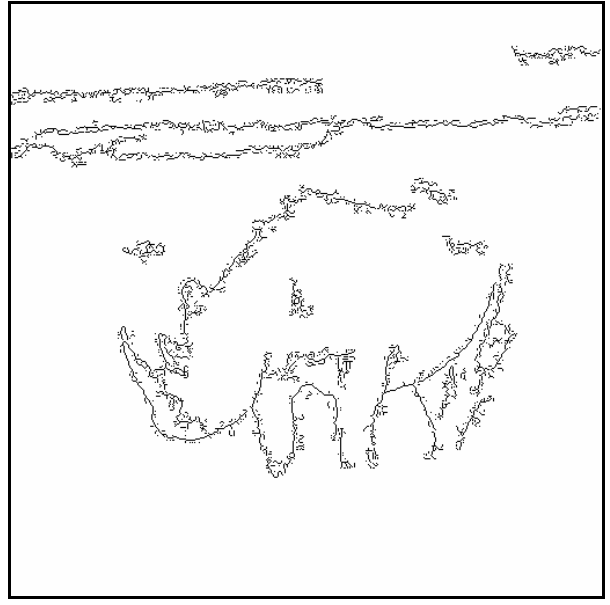

(b)

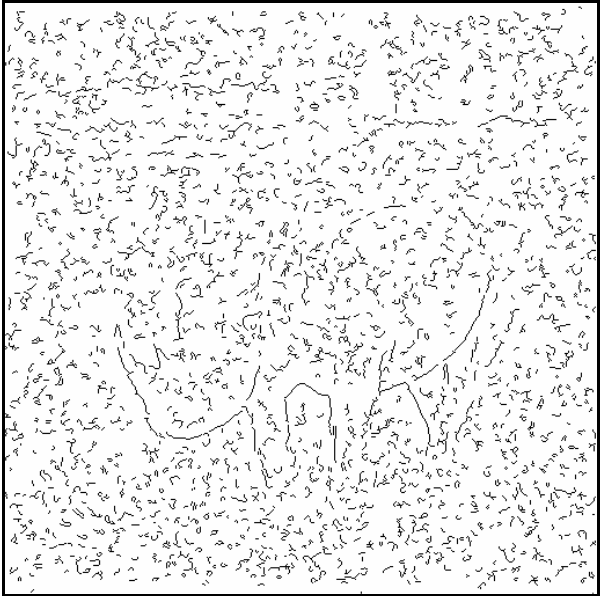

$(d)$

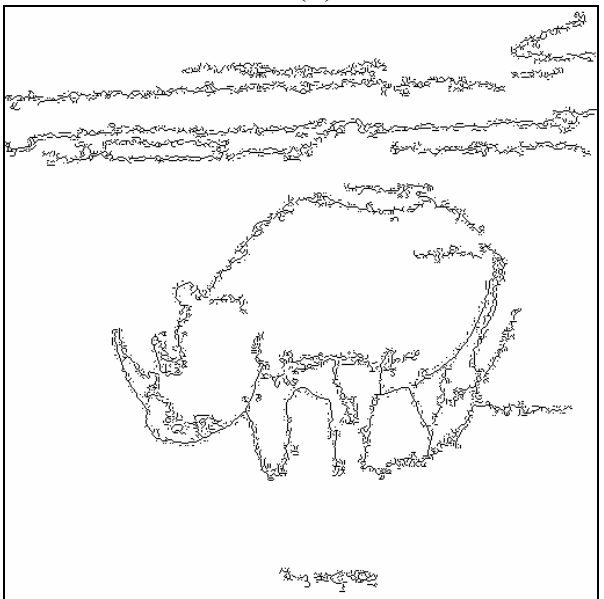

$(f)$

Fig. 9. (a). Input image (Rino) contaminated by additive Gaussian noise ( $\mathrm{SNR}=13.5 \mathrm{~dB}$ ). (b) Contours detected using the proposed approach. (c) Result of single scale Canny edge detection. (d) Result of single scale Canny edge detection augmented with surround inhibition. (e) Result of multiscale edge detection without surround inhibition. (f) Multiscale edge detection according to the scheme of Fig.4, where the inhibition step is not performed 


\section{EXPERIMENTAL RESULTS}

In this section some experimental results are presented. The test images "Elephant" and "Rino" are depicted in Fig. $6 a$ and Fig. $7 a$ respectively. In Fig. $6 b$ and Fig. $7 b$ the results of the proposed algorithm are given. As it is evident, the true contours are preserved and almost all the texture has been suppressed. For the sake of comparison, contours have been extracted from these test images using other approaches, such as the standard Canny edge detector (see Fig. $6 c$ and Fig. 7c) and the single scale Canny edge detector with surround inhibition as proposed in [18] (see Fig. $6 d$ and Fig. 7d). In Fig. $6 e$ and Fig. $7 e$, the results obtained using the CARTOON algorithm presented in [10] are given. In Fig. $6 f$ and Fig. $7 f$ results obtained by using the scheme presented in Fig.2 without making use of surround inhibition are depicted. It is evident from these experimental results that our approach gives the best performance among the considered approaches. Some more results, obtained for input image that are contaminated by Gaussian additive noise (SNR of 13.5 dB), are shown in Figs. 8 and 9. As evident from the experimental results the approach we propose is able to extract the image contours even in presence of severe noise, thus outperforming the other approaches used for comparison.

\section{CONCLUSIONS}

In this contribution we have proposed a contour detection and texture suppression. The presented method relies on a biologically motivated texture suppression technique, namely surround inhibition, in a multiscale framework. Multiresolution analysis reveals that at coarser scale, texture disappears, but some details about the true contours are also lost, since contours can be slightly shifted and junctions are destroyed. However, by proper combining the information at different scales, it is possible to obtain detailed and well localized contours and, at the same time, to suppress texture.

Our experiments show that surrounding inhibition and multiscale analysis, though driven by the same task, that is texture suppression, are quite orthogonal each other and that their combination produces better results than each one applied separately. Our experiments also show that the proposed method is robust to severe additive noise contamination.

\section{REFERENCES}

1. J.F. Canny, A computational approach to edge detection, IEEE Trans. Pattern Analysis and Machine Intelligence, Vol. 8 (6), 679-698, 1986.

2. W. Frei and C. Chen. Fast boundary detection: A generalization and a new algorithm. IEEE Trans. Computers, Vol. 26 (2) 988-998, 1977.

3. E.C. Hildreth. The detection of intensity changes by computer and biological vision systems. Computer Vision, Graphics and Image Processing, Vol. 22, 1--27, 1983.

4. J.-B. Martens. Local orientation analysis in images by means of the Hermite transform. IEEE Trans. Image Processing, Vol.6 (8) 1103--1116, 1997.

5. S. Ghosal and R. Mehrotra. Detection of composite edges. IEEE Trans. Image Processing, Vol. 3 (1) 14-25, 1994.

6. R.M. Haralick. Digital step edges from zero-crossings of second directional derivatives. IEEE Trans. Pattern Analysis and Machine Intelligence, Vol. 6 (1) 58-68, 1984.

7. T. Folsom and R. Pinter. Primitive features by steering, quadrature and scale. IEEE Trans. Pattern Analysis and Machine Intelligence, Vol. 20 (11), 1161-1173, 1998.

8. M.C. Morrone and R.A. Owens. Feature detection from local energy. Pattern Recognition Letters, Vol. 6, 303-313, 1987.

9. D. Ziou and S. Tabbone. Edge detection techniques - An overview. Int. J. Pattern Recognition and Image Analysis, Vol. 8 (4), 537-559, 1998.

10. W. Richards, H.K. Nishihara, and B. Dawson, CARTOON: A biologically motivated edge detection algorithm. MIT AI Memo 668 (1982). Also Chapt. 4 in W. Richards (ed.), Natural Computation, MIT Press, 1988, pages 5569.

11. M.J. Black, G. Sapiro, D. Marimont, and D. Heeger. Robust anisotropic diffusion. IEEE Trans. Image Processing, Vol. 7 (3), 421-432, 1998.

12. P. Perona and J. Malik. Scale-space and edge detection using anisotropic diffusion. IEEE Trans. Pattern Analysis and Machine Intelligence, Vol. 12 (7), 629-639, 1990. 
13. C. Grigorescu, N. Petkov, and M.A. Westenberg. Contour detection based on nonclassical receptive field inhibition. IEEE Trans. Image Processing, Vol. 12 (7), 729-739, 2003.

14. N. Petkov and P. Kruizinga. Computational models of visual neurons specialised in the detection of periodic and aperiodic oriented visual stimuli: bar and grating cells. Biological Cybernetics, Vol. 76 (2) 83-96, 1997.

15. N. Petkov and M.A. Westenberg. Supression of contour perception by band-limited noise and its relation to nonclassical receptive field inhibition. Biological Cybernetics, Vol. 88 236-246, 2003.

16. M.K. Kapadia, G. Westheimer, and C.D. Gilbert. Spatial distribution of contextual interactions in primary visual cortex and in visual perception. J. Neurophysiology, Vol. 84 (4), 2048-2062, 2000.

17. H.E. Jones, K.L. Grieve, W. Wang, and A. M. Sillito. Surround suppression in primate V1. J. Neurophysiology, Vol. 86 (10), 2011-2028, 2001.

18. C. Grigorescu, N. Petkov and M. A. Westenberg: Contour and boundary detection improved by surround suppression of texture edges, Image and Vision Computing, 22 (8), 2004, 609-622.

19. Ding, A; Goshtasby , A. On the Canny edge detector. Pattern Recognition, vol-34, pp.721-725, 2001

20. K.H. Liang, T. Tjahjadi and Y.H. Yang. "Bounded Diffusion for Multiscale Edge Detection Using Regularized Cubic B-Spline Fitting." IEEE Trans. On Systems, Man and Cybernetics, Vol. 29, No. 2, 1999, pp. 291-297.

21. H. J. A. M. Heimans, Morphological Image Operators. Boston: Academic Press, 1994.

22. H. J. A. M. Heimans, "Connected morphological operators for binary images”, Comp. Vis. Image Inderstand., vol. 73, pp. 99-120, 1999.

23. S.M. Konishi, A.L. Yuille, and J.M. Coughlan. "A Statistical Approach to Multi-Scale Edge Detection". Image and Vision Computing. Vol. 21/1.pp. 37-48, 2003.

24. S. Mallat, "A theory for multiresolution signal decomposition : the wavelet representation", IEEE Transaction on Pattern Analysis and Machine Intelligence, vol. 11, p. 674-693, July 1989.

25. [Galli1931]. A. Galli and A. Zama, "Untersuchungen über die Wahrnehmung ebener geometrischer Figuren, die ganz oder teilweise von anderen geometrischen Figuren verdeckt sind", Zeitschrift für Psychologie, Vol. 31, p. 308 348, 1931.

26. [Kanizsa1979]. G. Kanizsa, Organization in Vision, Essays on Gestalt Perception, New York: Praeger, 1979.

27. [Nothdurft1991]. H.C. Nothdurft, "Texture segmentation and pop-out from orientation contrast", Vision Research, Vol. 31, p. 1073-1078, 1991.

28. [Knierim1992] J.J. Knierim and D.C. van Essen, "Neuronal responses to static texture patterns in area V1 of the alert macaque monkey”, J. Neurophysiology, Vol. 67, p. 961-980, 1992.

29. [Nothdurft1999] H.C. Nothdurft, J. Gallant, and D.C. van Essen, "Response modulation by texture surround in primate area V1: Correlates of "popout" under anesthesia”, Visual Neuroscience, Vol. 16 (1), p. 15-34, 1999. 